Article available at http://www.parasite-journal.org or nttp://dx.dol.org/10.1051/parasite/1999062163

\title{
Neopsylla musseri N. SP. (Siphonaptera-Ctenophthalmidae), puce nOUVelle du SulaWesi Central (INDONÉSIe)
}

\author{
BEAUCOURNU J.C.* \& DURDEN L.A.**
}

Summary : NEOPSYLLA MUSSERI N. SP. (SIPHONAPTERA-CTENOPHTHALMIDAE) A NEW FLEA FROM CENTRAL SUlaWesi (INDONESIA)

This flea is a montane flea, known from elevations of 1,700-

2,300 $\mathrm{m}$, that parasitizes endemic murid rodents. It represents a newly recorded genus and family of fleas for Sulawesi.

Morphologically, the closest taxon to $N$. musseri n. sp. is $N$. sondaica Jordan, 1931 from Java; it is not closely related to $N$. luma Traub, 1954 from Borneo.

KEY WORDS : Neopsylla musseri n. sp., Siphonaptera, Ctenophthalmidae, Muridae, Sulawesi.

\section{Résumé :}

Cette puce, orophile (de 1700 à 2300 m), est parasite de muridés endémiques. Elle fournit à la faune de cette région un genre nouveau et une famille nouvelle. Le taxon,

morphologiquement le plus apparenté, est $\mathrm{N}$. sondaica Jordan, 1931 de Java; il n'est pas directement affilié avec N. luma Traub, 1954 de Bornéo.

MOTS CLÉS : Neopsylla musseri n. sp., Siphonaptera, Ctenophthalmidae, Muridae, Sulawesi.

\section{INTRODUCTION}

4 n 1975, le Dr. Guy Musser (Department of Mammalogy, American Museum of Natural History, New York) a collecté des mammifères dans le Sulawesi Central et plus précisément sur le Gunung Nokilalaki. Au cours de cette mission des siphonaptères furent récoltés : 19 d'entre eux (13 mâles et 6 femelles) représentent une espèce nouvelle appartenant au genre Neopsylla Wagner, 1903 (Ctenophthalmidae, Neopsyllinae), que nous décrivons ici. Les autres exemplaires sont : - une femelle de Macrostylophora Ewing, 1929 (Ceratophyllidae, Ceratophyllinae), genre et sous-famille non signalés antérieurement du Sulawesi (l'espèce semble également inédite). Traub (1972) écrivait : "Macrostylophora undoubtedly infests squirrels in Sarawak despite the dearth of reports and it would not be surprising if it accompanied Callosciurus prevosti and C. notatus to Celebes". Musser (1987) considère ces deux écureuils comme introduits, mais d'autres Sciuridae (six espèces et trois genres) sont endémiques. - des représentants du genre Sigmactenus Traub, 1950 (Ceratophyllidae, Leptopsyllinae) et des Pygiopsyllidae.

\footnotetext{
* Laboratoire de Parasitologie \& Zoologie appliquée, Faculté de Médecine, Avenue du Prof. Léon Bernard, 35043 Rennes Cedex, France.

** Institute of Arthropodology \& Parasitology, Georgia Southern University, P.O. Box 8056, Statesboro, Georgia 30460, USA. Correspondance : J.C. Beaucournu.

Tél. : 0299336959 - Fax : 0299336941

E-mail : jbeaucou@univ-rennes1.fr
}

\section{NEOPSYLLA MUSSERI N. SP.}

\section{MATÉRIEL DE DESCRIPTION}

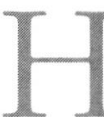
olotype mâle, sur Bunomys penitus (Miller \& Hollister, 1921) (préparation B 94845), Indonésie, Central Sulawesi, Gunung Nokilalaki $\left(120^{\circ} 10 \mathrm{E}, 1^{\circ} 16 \mathrm{~S}\right), 2100 \mathrm{~m}, 16$ mars 1975 (G.G. Musser rec.); Allotype femelle sur Paruromys dominator (Thomas, 1921) (B 94846), do, 2050 m, 21 mars 1975.

Paratypes

- Sur B. penitus, $1 \mathrm{~m}, d^{\circ}, 1850 \mathrm{~m}, 23$ février 1975 (B 94827); $3 \mathrm{~m}, 2 \mathrm{f}, d^{\circ}, 1830 \mathrm{~m}, 24$ février 1975 (B 94826, B 94829; deux hôtes, quatre préparations); $1 \mathrm{f}, d^{\circ}, 1975 \mathrm{~m}, 24$ février 1975 (B 94828); $1 \mathrm{~m}, 1 \mathrm{f}$, $d^{\circ}, 1740 \mathrm{~m}, 26$ février 1975 (B 94831); $1 \mathrm{~m}, d^{\circ}, 2210 \mathrm{~m}$, 27 février 1975 (B 94834); $1 \mathrm{~m}, d^{\circ}, 2300 \mathrm{~m}, 4$ mars 1975 (B 94862); $1 \mathrm{~m}, 1 \mathrm{f}, d^{\circ}, 2210 \mathrm{~m}, 4$ avril 1975.

- Sur P. dominator, $1 \mathrm{~m}, d^{\circ}, 2300 \mathrm{~m}, 12$ mars 1975 (B 94843); $1 \mathrm{~m}, d^{\circ}, 2070 \mathrm{~m}, 14$ mars 1975 (B 94840). - sur Maxomys musschenbroekii (Jentink, 1878), 2 m, do, $1740 \mathrm{~m}, 25$ février 1975 (B 94830, deux préparations).

Ce matériel nous a été confié pour étude déjà monté. Les mâles des préparations B 94829, B 94843 et B 94855 avaient été partiellement disséqués pour prélèvement et montage à part du tergite IX gauche, ce qui a entraîné quelques lésions des segments, ou du sternite IX, adjacents. 
Hôtes

Les hôtes ayant livré ces puces sont tous des Rongeurs Muridés (Murinés) endémiques du Sulawesi (Musser \& Carleton, 1993) : Bunomys penitus se rencontre dans les forêts orophiles à importante pluviométrie dans le Centre et le Sud-est du Sulawesi; Paruromys dominator et Maxomys musschenbroekii se trouvent sur l'ensemble du territoire et seraient limités aux zones d'altitude (Musser, 1987), toutefois Durden (1986) signale M. musschenbroekii à partir de $220 \mathrm{~m}$.

Les puces furent capturées de 1700 à 2300 m, bien que les piégeages furent commencés à plus basse altitude. Les séries de végétation sont les suivantes :

- de 1600 à 1800 m (forêt humide de moyenne altitude), le "chataignier" Castanopsis acuminatissima est l'arbre dominant mais la cohorte comprend également Agathis sp., Elaeocarpus sp., des magnolias (Aromadendron spp.), des conifères (Dacrydium spp. et Podocarpus spp.), divers "chênes" (Lithocarpus spp.), l'érable Acer caesium et l'endémique Macadamia bildebrandii. - de 1800 à 2200 m, la végétation dominante est celle de forêt humide de haute altitude avec des arbres de petite taille, couverts de mousse, les feuillus sont peu élevés, bien que la canopée culmine cependant à 10$20 \mathrm{~m}$. Les grands arbres ont perdu leurs contreforts à la base de leurs troncs; les épiphytes manquent, ainsi que les plantes adventices ce qui donne un milieu assez ouvert. Les arbres présents sont Lithocarpus, qui est dominant, mais aussi, inter alia, des "lauriers" (Litsea et Crytocarya), des magnolias, des "noyers" (Engelhardtia), des conifères et de rares "châtaigniers". - de $2200 \mathrm{~m}$ au sommet ( ca 2280 m), des conifères (Dacrydium sp.) de petite taille (3 à $5 \mathrm{~m}$ ) prédominent. Ils sont accompagnés de divers arbustes buissonnants tels que Rhododendron. Les mousses "géantes", Spiridens et Dawsonia pendent des arbres; des sphaignes (Sphagnum), des népenthès, des fougères, ... couvrent le sol.

Les températures moyennes diurnes vont de $18^{\circ} 5 \mathrm{C}$ à $13^{\circ} \mathrm{C}$ dans la zone de récoltes de Neopsylla (soit de 1700 à $2300 \mathrm{~m}$ ). L'hygrométrie relative varie de 85 à 100 \% (Musser \& Dagosto, 1987; Whitten et al., 1987; Durden, obs. pers.).

\section{Derivatio nominis}

Cette espèce est dédiée à son collecteur, notre collègue et ami le Dr. Guy G. Musser, mammalogiste de réputation mondiale et spécialiste, entre autres, de la faune du Sulawesi.

\section{Dépôt des types}

Holotype et Allotype sont déposés à l'US National Museum (Smithsonian Institution) à Washington. Les paratypes sont répartis dans les collections des auteurs (ultérieurement, pour celle du premier signataire, au
Laboratoire d'Entomologie du Museum National d'Histoire Naturelle de Paris), dans celles du Museum Zoologicum Bogoriense à Bogor (Indonésie), de l'American Museum of Natural History de New York et de la Medical and Veterinary Division, Natural History Museum, Londres.

\section{DESCRIPTION}

Neopsylla appartenant au groupe stevensi, sensu Hopkins \& Rothschild (1962) et Liu et al. (1986).

Capsule céphalique : tubercule frontal petit. Epine antérieure (ou externe) de la cténidie génale acuminée, faisant la moitié ou un peu plus de la longueur de l'épine postérieure qui est, relativement, fine. Palpe labial long, atteignant ou dépassant les 3/4 de la longueur de la coxa I ; segment apical (V) aussi long, ou un peu plus long, que les deux segments précédents (III et IV); ratio (de I à V) : 1-0,85-0,75-0,75-1,65. Rangée frontale supérieure de sept soies (rarement six, voire cinq), bien développées chez les mâles, plus courtes chez les femelles. Quatre soies dans la rangée préoculaire. Trois rangées de soies occipitales de respectivement : chez les mâles, 3 à 5,4 à 6,5 ou 6 ; chez les femelles, 4 à 7,6 ou 7,6 ou 7 .

Thorax : une rangée de 6 ou 7 soies sur le prothorax, précédée d'un rang vestigial de 0 à 2 petites soies chez les mâles, 2 à 6 chez les femelles. Cténidie pronotale de 22 épines (24 chez un mâle) ; épines dorsales légèrement plus longues que le pronotum chez les mâles, un peu plus courtes ou de même longueur chez les femelles. Métépimeron portant de 7 à 10 soies chez les mâles, 9 à 10 (10: 5 fois sur 6) chez les femelles. Tibia III : 9 (plus rarement 10) encoches sur sa marge postérieure, dont la sétation normale (et particulièrement celle de l'holotype) est : 1, 2, 1, 2, 1, 2, 1, 1, 4, le chiffre indiquant le nombre de soies par encoche. Sétation tarsale : tarses I et II, 5 soies latérales; tarse III, 4 soies. Abdomen, segments non modifiés : pas de sclérification dorsale des tergites. Spinules présentes sur les tergites I à V, bien que deux mâles montrent, unilatéralement une spinule sur t. VI. Leur nombre est le suivant (de I à V) pour un côté. Mâles : 1 ou 2 (2 le plus souvent), 2 (1 exemplaire en a 4 unilatéralement), 2 (1 exemplaire n'en a qu'une, bilatéralement), 1 ou 2 (le plus souvent 1), 1 : Femelles : 1 à 3, 2 (un spécimen n'en montre qu'une d'un côté), 1 ou 2, 1 (0 unilatéralement chez un exemplaire), 0. Stigmates acuminés postérieurement.

Abdomen, segments génitaux mâles et phallosome. Sternite VIII (Fig. 1), caractérisé par un prolongement inféro-apical plus long que haut; sinon le segment dans son ensemble est plus haut que long à bord postérieur subrectiligne ou doucement concave jusqu'à une petite encoche surplombant le prolongement. Segment IX (Fig. 2) : lobe antérieur du basimère $\left(\mathrm{L}_{1}\right)$ à apex arrondi, moins de deux fois plus haut que large. Lobe 

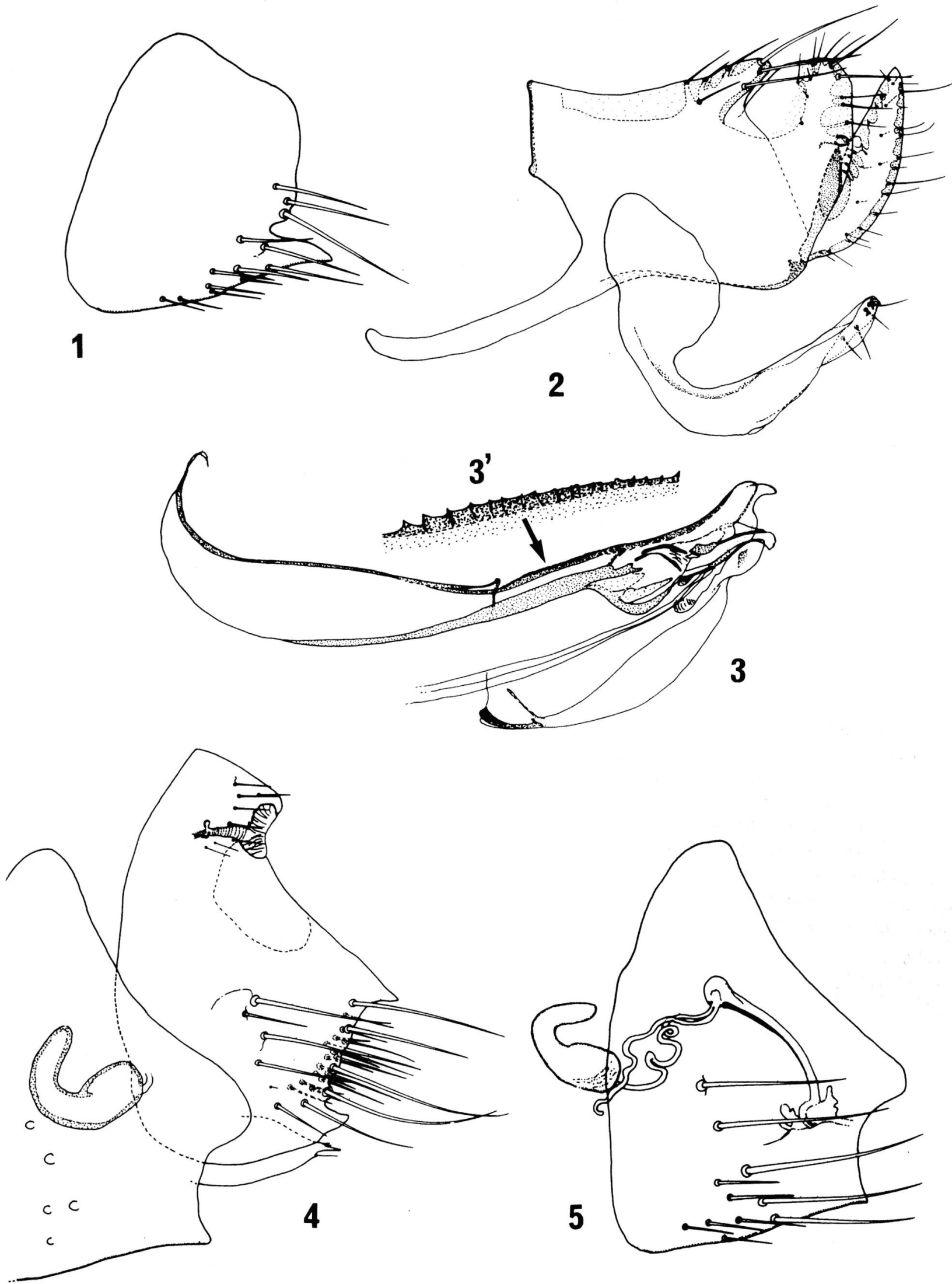

Figs. 1-5. - Neopsylla musseri n. sp. 1 : sternite VIII, holotype mâle. 2 : segment IX, holotype. 3 : phallosome, holotype (3' : segment agrandi du lobe dorso-médian de la lamina media). 4 : sternite VII, segment VIII, spermathèque, allotype femelle. 5: sternite VII, spermathèque et ducti, paratype femelle. 
ventral (ou postérieur, ou $\mathrm{L}_{2}$ ) large; le sinus le séparant de $\mathrm{L}_{1}$ est étroit et profond mais toute la partie antéro-dorsale de ce lobe est peu sclérifiée rendant difficile, à faible grandissement, l'appréciation des contours respectifs de ces deux lobes. Fovea située, approximativement, aux $2 / 3$ supérieurs de la marge postérieure. Télomère plus court que cette marge : sa base est à distance de l'angulation ventrale du basimère ; apex acuminé atteignant le sommet du basimère ; soies marginales relativement longues, mais fines. La soie marginale inférieure la plus longue s'insère au niveau de l'acetabulum. Sternite IX : bras proximal et bras distal sensiblement de même longueur. Bras proximal à zone apicale environ deux fois plus haute que large, sans processus postérieur saillant. Bras distal diminuant progressivement de largeur; apex portant 2 à 5 petites soies spiniformes non pigmentées et quelques autres soies plus longues et fines. Phallosome (Fig. 3) : Lamina media se terminant antérieurement par un long et fin processus sclérotisé puis membraneux ; présence d'un éperon proximal de l'aedeagus, court mais nettement indiqué; lobe dorso-médian fortement sclérotisé et strié latéro-latéralement donnant à cette zone un aspect échinulé en coupe optique (Fig. 3'). Base antéro-ventrale de la paroi aedeagale très fortement sclérotisée et pigmentée. Tubus interior doucement arqué ventralement à l'apex; paroi dorsale de celui-ci portant une nette expansion triangulaire. Hamulus en oval allongé. Segments génitaux femelles, spermathèque et ducti (Figs. 4-5). Sternite VII caractérisé par un large lobe arrondi sur le bord distal, surplombant une marge concave; 9 à 11 soies latérales dont 4 à 5 grandes. Tergite VIII : fosse spiraculaire relativement grande ; bord postéro-apical rectiligne entre 2 lobes triangulaires, l'un dorsal, l'autre ventral ; sétation externe comportant 4 grandes soies marginales et 5 à 6 latérales; sétation interne, marginale ou submarginale, comportant une quinzaine de soies. Sternite VIII large sauf à l'apex qui est très acuminé. Spermathèque : bulga plus longue que large à bords dorsal et ventral parallèles, plus ou moins quadrangulaire et non ovale; billa longue. Ductus bursae, long, doucement arqué, à bord antéro-ventral sclérotisé; une petite sclérification, plus ou moins nette, est visible à la jonction du bord postéro-dorsal et de l'ostium bursae. Ductus spermathecae et ductus obturatus sont dans un rapport de longueur d'environ $1: 0,75$. Dimensions (insectes montés) : mâles, 2,2 à 2,5 (holotype : 2,4); femelles, 2,8 à $3,2 \mathrm{~mm}$ (allotype : 3,1).

\section{DISCUSSION}

$\mathrm{L}$ a récolte de Neopsylla musseri n. sp. au Sulawesi est intéressante à plus d'un titre. C'est la première mention de ce genre sur l'ile mais aussi celle du premier Ctenophthalmidae. Par ailleurs, c'est la station la plus extrême connue pour ce genre (Holarctique et Oriental), ou même le genre voisin Rothschildiana Smit, 1952, ce dernier étant seulement signalé de Java et de Malaisie occidentale (ou péninsulaire).

Les Neopsylla de la zone orientale appartiennent soit au groupe stevensi $\mathrm{s}$. str; soit au groupe anoma, sensu Liu et al., 1986, caractérisé entre autres par ses stigmates abdominaux arrondis postérieurement. Toutes les espèces connues du Sud-Est asiatique sont parasites de Murinés (Traub, 1972) et ceci est confirmé tant par le présent travail que par au moins l'une des deux espèces récemment décrites du Vietnam (Beaucournu \& Sountsov, 1997), l'hôte très aberrant de la seconde (Beaucournu \& Sountsov, sous presse), l'insectivore Hylomys sinensis Trouessart, 1909, étant vraisemblablement accidentel.

A l'heure actuelle, 10 taxa sont connus de cette région (l'un, signalé, par Traub (1972) n'est pas décrit) :

- N. stevensi stevensi Rothschild, 1915 : Vietnam-Nord, - N. sondaica Jordan, 1931 : Java,

- N. avida Jordan, 1931 : Vietnam-Sud,

- N. tricata Jordan, 1931 : Vietnam-Sud,

- N. dispar Jordan, 1932 : Malaisie péninsulaire, Myanmar (ex Birmanie), Thailande,

- N. luma Traub, 1954: Bornéo (Sabah),

- N. bana Beaucournu \& Sountsov, 1997 : VietnamSud,

- N. ninae Beaucournu \& Sountsov, 1999 : VietnamNord,

- N. musseri Beaucournu \& Durden, présent travail : Sulawesi,

- N. n. sp. (in Traub, 1972) : Java.

Sur le plan morphologique, et sans doute phylétique, $N$. musseri n'est pas directement affiliée avec N. luma qui est d'ailleurs, d'après les dessins de Traub (1954, Figs. 15 et 19), du groupe anoma. N. sondaica en est plus proche par le segment IX du mâle, mais le sternite VII de celui-ci, comme le sternite VII de la femelle sont totalement différents.

\section{REMERCIEMENTS}

I e travail de terrain de Guy Musser au Sulawesi fut financé par le "Celebes Fund of the American Museum of Natural History (AMNH)" et par les "Archbold Expeditions, Inc.". La mission de Lance Durden au Sulawesi fut prise en charge par le "Committee for Research and Exploration of the National Geographic Society", Washington DC; ses séjours à l'AMNH afin de collecter les ectoparasites par l'étude des peaux de rongeurs collectés, furent financés par le "Celebes Fund of the AMNH". 


\section{RÉFÉRENCES}

Beaucournu J.C. \& Sountsov V.V. Puces du genre Neopsylla collectées au Vietnam : description de $N$. bana $\mathrm{n}$. sp. (Siphonaptera, Ctenophthalmidae). Bulletin de la Société entomologique de France, 1997, 102, 205-209.

Beaucournu J.C. \& Sountsov V.V. Puces nouvelles ou peu connues du Vietnam; Ctenophthalmidae et Pygiopsyllidae (Insecta-Siphonaptera). Bulletin de la Société entomologique de France, 1999, 104, 77-83.

Durden L.A. The reinfestation of forest rats (Maxomys musschenbroekii) by epifaunistic arthropods in Sulawesi, Indonesia. Journal of Tropical Ecology, 1986, 2, 283-286.

Hopkins G.H.E \& Rothschild M. An illustrated catalogue of the Rothschild collection of fleas (Siphonaptera) in the British Museum (Natural History). Vol. III : Hystrichopsyllidae (Acedestiinae, Anomiopsyllinae, Hystrichopsyllinae, Neopsyllinae, Rhadinopsyllinae and Stenoponinae). 1962, British Museum, 560 pp., 1 carte, 10 pl.

Liu Zinying et al. Fauna sinica, Insecta, Siphonaptera. 1986, Science Press, Bejing, XXIII + 1334 pp. (en chinois, résumé anglais).

Musser G.G. The mammals of Sulawesi, pp. 73-93, in Biogeographical evolution of the Malay Archipelago, 1987, (T.C. Whitmore, ed.), Oxford University Press, Oxford, $147 \mathrm{pp}$.

Musser G.G. \& Carleton M.C. Family Muridae, pp. 501-755, in Wilson D.E. \& Reeder D.M. (ed.) : Mammal species of the World - a taxonomic and geographic reference, 1993,

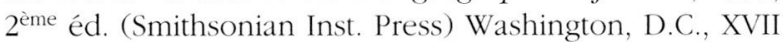
$+1206 \mathrm{pp}$.

Musser G.G. \& Dagosto M. The identity of Tarsius pumilus, a pygmy species endemic to the montane mossy forests of central Sulawesi. American Museum Novitates, 1987, $2867,1-53$.

Traub R. Malaysian Parasites XI : Sigmactenus alticola and Neopsylla luma, new species of fleas from North Borneo. Studies from the Institute of Medical Research, Malaya. Singapour, 1953 (paru 1954), 26, 184-194.

Traub R. The Gunong Benom Expedition 1967. 13. Notes on Zoogeography, convergent evolution and taxonomy of fleas (Siphonaptera), based on collections from Gunong Benom and elsewhere in South-East Asia. III. Zoogeography. Bulletin of the British Museum (Natural History), Zoology, 1972, 23, 391-450.

Whitten A.J., Mustafa M. \& Henderson G.S. The ecology of Sulawesi, 1987, Gadjah Mada University Press, Yogyakarta, XXI + 777 pp.

Reçu le 9 janvier 1999

Accepté le 25 mars 1999 